\title{
Searches for low-mass neutral Higgs bosons using the CMS detector
}

\author{
Camilo Carrillo*t \\ Université Claude Bernard-Lyon I (FR) \\ E-mail: carrillo@cern.ch
}

\begin{abstract}
A summary of the searches performed by the CMS experiment on the $8 \mathrm{TeV}$ LHC data for direct production of additional scalars and pseudo-scalar neutral bosons with masses below the higgs boson $\mathrm{h}_{125}$ is presented. These searches are motivated by the predictions of several BSM theories, most significantly extensions of the non minimal extensions of the MSSM like the NMSSM, where additional scalar and pseudo-scalar states are expected. The mass range from $350 \mathrm{MeV}$ to $110 \mathrm{GeV}$ is explored with different final states. The current status and the extension of these searches to the run-II of the LHC are described.
\end{abstract}

38th International Conference on High Energy Physics

3-10 August 2016

Chicago, USA

${ }^{*}$ Speaker.
${ }^{\dagger}$ on behalf of the CMS collaboration

(C) Copyright owned by the author(s) under the terms of the Creative Commons 


\section{Introduction}

The observation of a new Higgs-like particle in 2012 [1] is already a nostalgic memory, this observation just closed a chapter in the construction of the SM (Standard Model of particles). There is not yet explanation for several important features of nature such as the hierarchy problem or the nature of dark matter [2]. It is expected that new particles exist in nature in order to understand this unexplained phenomena. But the hypothetical existence of new particles arises new questions: Is the $125 \mathrm{GeV}$ scalar [3] [4] [5] really the Standard Model Higgs Boson?, Is it the lightest?. Some BSM theories predict modified and extended Higgs sectors, possibly with additional low-mass $\left(\mathrm{m}_{h}<125 \mathrm{GeV}\right)$ scalars / pseudo-scalars. The most representative models addressing these questions with its list of main features are:

- General 2HDM [6]:

- 2 Higgs doublets $\rightarrow 5$ Higgs bosons : $h, H, a, H^{ \pm}$

- 4 types, main parameters : $\tan \beta, \alpha$

- compatible with a $125 \mathrm{GeV}$ SM-like scalar $(h$ or $H)+$ a light Higgs Boson $(a$ or $h)$

- MSSM [7] :

- minimal supersymmetric model (2HDM type II)

- low-mass (pseudo) scalars disfavored within this model

- NMSSM [8] [9] :

- 2 Higgs doublets +1 singlet $\rightarrow 7$ Higgs bosons $: h_{1}, h_{2}, h_{3}, a_{1}, a_{2}, H^{ \pm}$

- solves the " $\mu$-problem" of the MSSM

- compatible with a $125 \mathrm{GeV}$ SM-like scalar $\left(h_{1}\right.$ or $\left.h_{2}\right)$ + a mostly "singlet-like" light Higgs Boson $\left(a_{1}\right.$ or $\left.h_{1}\right)$

Other BSM theories also motivate the search for a low-mass Higgs boson, such as dark-SUSY models [10] or general $2 \mathrm{HDM}+\mathrm{S}$ [11]. In this context the searches for direct production of a low mass neutral higgs boson, in 3 decay channels, done by the CMS experiment [12] are described and the results obtained are shown.

\section{Low mass neutral higgs boson, results}

\subsection{The diphoton channel $h \rightarrow \gamma \gamma$}

Low mass scalars decaying into two photons can be accommodated in 2HDM and NMSSM models. For some benchmark points it is allowed the existence of such a particle with a sizable cross-section. The product of the cross-section and the branching-ratio of the diphoton channel could reach up to 3.5 times the prediction of the standard model [13]. This analysis [14] extends the reach of the tools developed for the discovery of the Higgs Boson in 2012 to lower mass range, 
between 80 and $110 \mathrm{GeV}$. If a new boson with the described characteristics exist, it should be visible as an excess of events localized in a narrow peak over a smoothly falling background, composed by prompt photons and jets misidentified as photons. The probability to get Drell Yan electrons reconstructed as photons after the event selection is reduced by applying an electron veto. However a contribution from this type of electrons is expected and observed in the data analyzed around the $\mathrm{Z}$ mass. The modeling of the $\mathrm{Z}$ background (misidentified electrons) is an important feature of this search.

In order to improve the sensitivity, the selected events are separated into four categories obtained from a multivariate classifier optimising Signal/Background from purity and mass resolution. The limits on the product of the cross-section times branching ratio are presented in 1 . No significant excess with respect to the expected background is observed. The observed limit ranges from $75 \mathrm{fb}$ at $80 \mathrm{GeV}$ mass hypothesis to $42 \mathrm{fb}$ at a $110 \mathrm{GeV}$ mass. The largest excess has a local significance of $1.9 \sigma$ for a mass of $98 \mathrm{GeV}$.

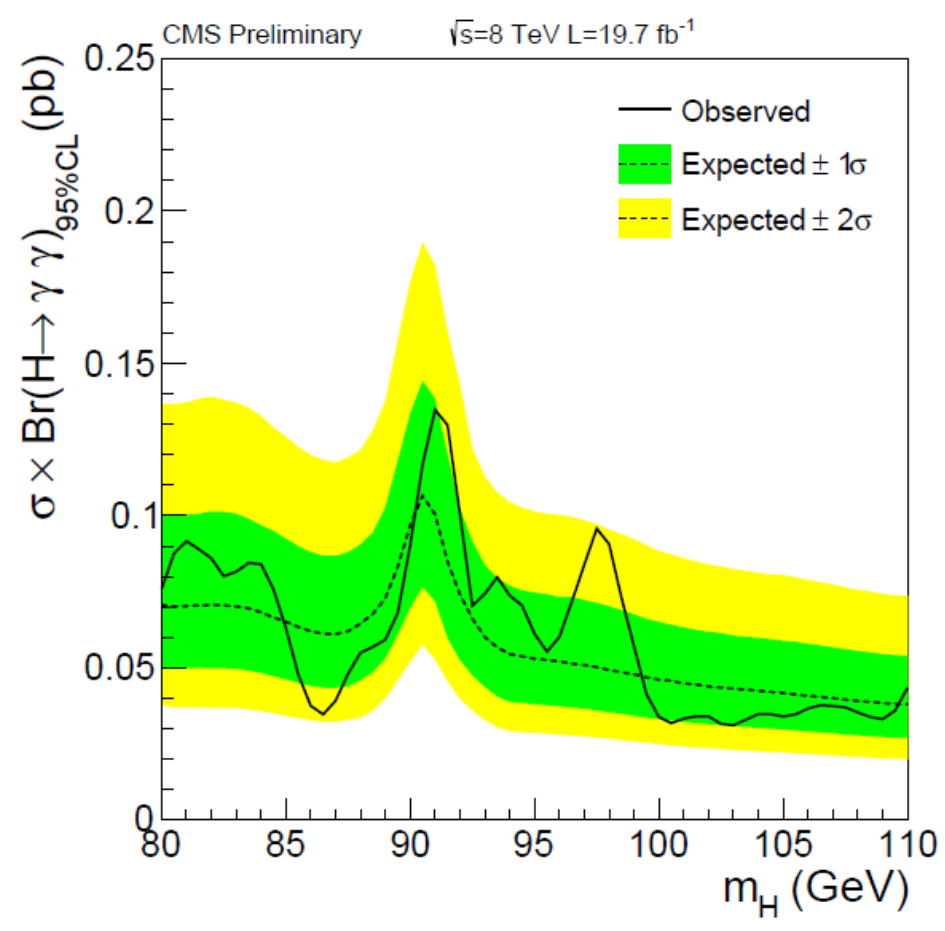

Figure 1: Exclusion limits at 95\% Confidence Level (asymptotic CLs approximation) on the product of the cross-section and branching ratio into two photons.

\section{$2.2 b \bar{b}$ associated production, bbA with $\mathbf{A} \rightarrow \tau \tau$}

In this analysis [15] the search for a light pseudo-scalar Higgs boson decaying to two $\tau$ leptons produced in association with a $b \bar{b}$ pair is performed. Three final states are considered: $\mu \tau_{h}{ }^{1}, e \tau_{h}$

\footnotetext{
${ }^{1} \tau_{h}$ represent the hadronic $\tau$ decay
} 
and $e \mu$. The $\mu \mu$ and $e e$ final states are not considered because of the small branching fractions and large backgrounds. Finally $\tau_{h} \tau_{h}$ is not considered because of inefficiencies due to the trigger thresholds. The pseudo-scalar boson mass probed ranges between 25 and $80 \mathrm{GeV}$.

The main backgrounds are $t \bar{t}, \mathrm{~W}+$ jets and QCD multi-jets. No evidence for a pseudo-scalar boson is found and upper limits on the production cross section times branching fraction are set for every studied channel as showing in fig. 2 .
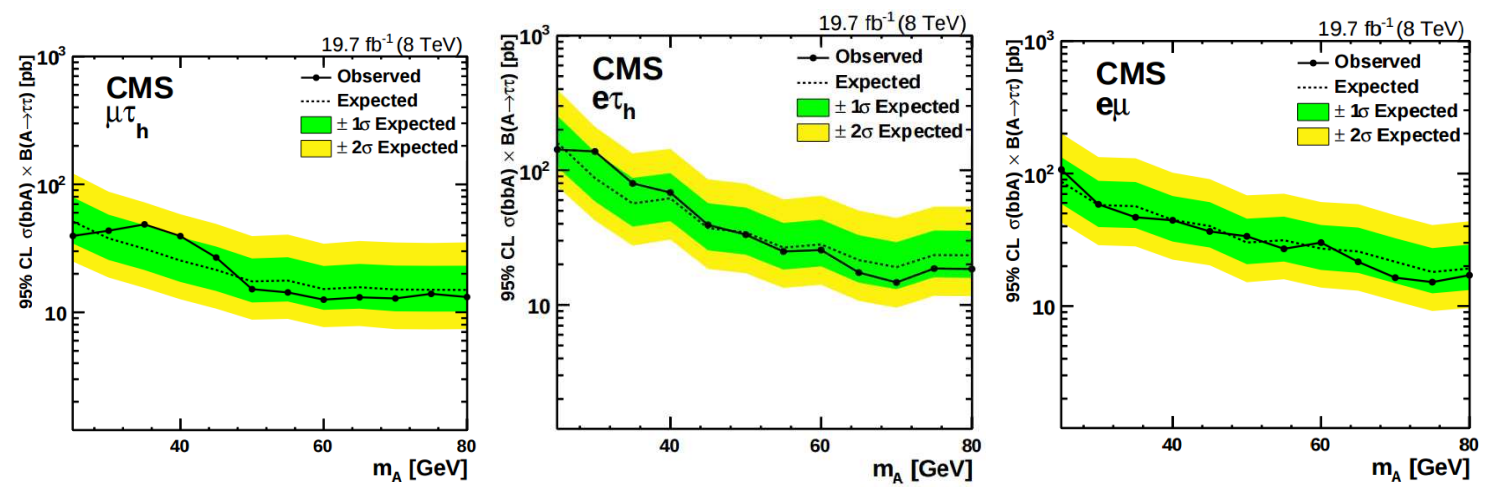

Figure 2: Expected cross sections for Type II 2HDM, superimposed on the expected and observed combined limits from the $\mathrm{a}+\mathrm{bb} \rightarrow \tau \tau+\mathrm{bb}$ searches.

\section{$2.3 h+X \rightarrow b \bar{b}+X$}

In this search [16] the decay of a low mass higgs boson to a pair of b quarks is performed. The theoretical context of this search is the NMSSM model in two benchmark points: the "modified P4" benchmark and the "decoupled squarks" scenario[19] [20]. The type of process considered is the pair production of colored supersymmetric partners, decaying to neutralinos and charginos, and subsequently into a light scalar $\mathrm{h}_{1}$ and the LSP. A high hadronic activity is expected due to the $\tilde{q}$ and $\tilde{g}$ decays to quarks. Because of this the final topology of the signal event is a pair of b quarks coming from the decay of $h_{1}$, large missing energy and at least two very energetic jets. Masses between 30 and $110 \mathrm{GeV}$ are probed. The signal model is composed by a model independent $h_{1}$ resonance and a model dependant SUSY excess. Limits are set on the cross-section of $h_{1}$ only production times the branching fraction into bb. These results are interpreted with the NMSSM benchmark scenarios. Limits are set on the NMSSM cross section times branching fraction into bb together with the prediction of the modified P4 scenario fig.3, and limits on NMSSM parameters in the decoupled squarks scenario are also shown.

\section{Conclusions}

A Comprehensive low-mass higgs searches have been performed in CMS with the LHC run-I data. A challenge for all these searches is the trigger and the reconstruction of low $p_{T}$ objects. Both direct (this talk) and pair production have been considered by CMS but no evidence for new particle production has been found. 2HDM and NMSSM interpretations have been evaluated and parameter space regions excluded and benchmark scenarios have been ruled out. Improvements 


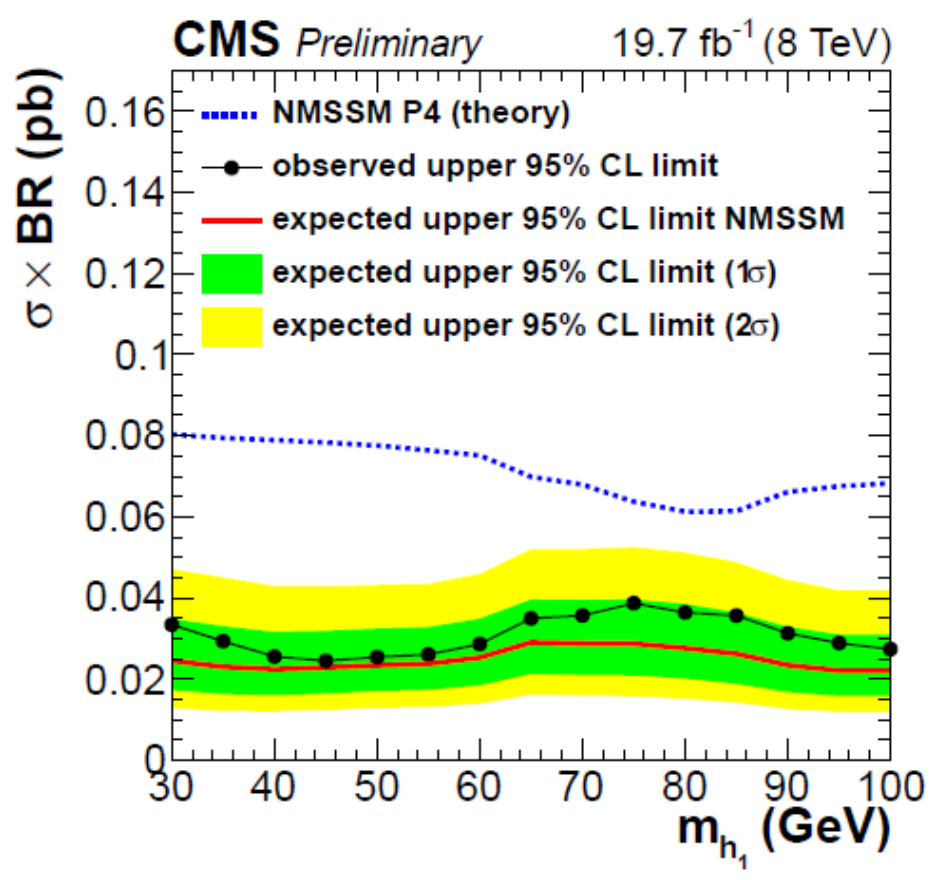

Figure 3: Limits for the light Higgs boson production cross section times branching ratio, for the $\mathrm{h}+\mathrm{X} \rightarrow$ $\mathrm{bb}+\mathrm{X}$ search in the NMSSM P4 benchmark.

like new triggers for even lower mass regions are already deployed during run-II and new final states, and production modes are under development.

\section{Acknowledgement}

The author is grateful to the LABEX Lyon Institute of Origins (ANR-10-LABX-0066) of the Université de Lyon for its financial support within the program "Investissements d'Avenir" (ANR11-IDEX-0007) of the French government operated by the National Research Agency (ANR).

\section{References}

[1] ATLAS Collaboration, Observation of a new particle in the search for the Standard Model Higgs boson with the ATLAS detector at the LHC, Physics Letters B 716 ( 2012 ) 1-29.

[2] Ellis, John, Limits of the standard model, arXiv preprint hep-ph/0211168 ( 2002 ).

[3] Englert, Francois and Brout, Robert, Broken symmetry and the mass of gauge vector mesons, Physical Review Letters 13 ( 1964 ) 321.

[4] ATLAS and CMS collaborations, Precise determination of the mass of the Higgs boson and tests of compatibility of its couplings with the standard model predictions using proton collisions at 7 and 8 TeV, The European Physical Journal. C, Particles and Fields 75 ( 2015 ).

[5] ATLAS Collaboration, Measurement of the Higgs boson mass from the $H \rightarrow \gamma \gamma$ and $H \rightarrow Z Z^{*} \rightarrow 4 l$ with the ATLAS detector using 25 fb- 1 of pp collision data, Physical Review D 90 ( 2014 ) 052004-1. 
[6] Branco, Gustavo Castelo and Ferreira, PM and Lavoura, L and Rebelo, MN and Sher, Marc and Silva, Joao P, Theory and phenomenology of two-Higgs-doublet models, Physics reports 516 ( 2012 ) $1-102$.

[7] Martin, Stephen P, A supersymmetry primer, Adv. Ser. Direct. High Energy Phys 21 ( 2010 ) $1-153$.

[8] Ellwanger, Ulrich, Higgs bosons in the next-to-minimal supersymmetric standard model at the LHC, The European Physical Journal C 71 ( 2011 ) 1-10

[9] Badziak, Marcin and Olechowski, Marek and Pokorski, Stefan, New regions in the NMSSM with a 125 GeV Higgs, arXiv preprint arXiv:1304.5437 ( 2013 ).

[10] Arkani-Hamed, Nima and Finkbeiner, Douglas P and Slatyer, Tracy R and Weiner, Neal , A theory of dark matter, Physical Review D 79 ( 2009 ) 015014.

[11] Chang, Spencer and Dermisek, Radovan and Gunion, John F and Weiner, Neal , Nonstandard Higgs boson decays, arXiv preprint arXiv:0801.4554 ( 2008 ).

[12] Adolphi, Roman and others, The CMS experiment at the CERN LHC, Jinst 803 ( 2008 ) S08004-2008 .

[13] Jia-Wei, Fan and others, Study of diphoton decays of the lightest scalar Higgs boson in the Next-to-Minimal Supersymmetric Standard Model, Chinese physics C 38 (2014) 073101.

[14] Search for new resonances in the diphoton final state in the mass range between 80 and $110 \mathrm{GeV}$ in pp collisions at $\sqrt{s}=8 \mathrm{TeV}$, institution $=C E R N$, author $=C M S$ Collaboration , address $=$ Geneva, number $=$ CMS-PAS-HIG-14-037, year $=2015$, reportnumber $=$ CMS-PAS-HIG-14-037, url = https://cds.cern.ch/record/2063739, tech. rep.

[15] CMS Collaboration, Search for a low-mass pseudoscalar Higgs boson produced in association with a b-bbar pair in pp collisions at sqrt $(s)=8 \mathrm{TeV}$, arXiv preprint arXiv:1511.03610 ( 2015 ).

[16] CMS Collaboration, Search for a light NMSSM Higgs boson produced in supersymmetric cascades and decaying into a b-quark pair, CMS PAS HIG-14-030, http://cds. cern. ch/record/2002557 ( 2015 ). 